\title{
ČECH-STONE REMAINDERS OF DISCRETE SPACES
}

\author{
PETER NYIKOS
}

\section{INTRODUCTION}

The study of Cech-Stone remainders has long been a major theme in settheoretic topology. A whole book [13] was published that primarily dealt with the remainder $\omega^{*}=\beta \omega-\omega$ of the countable discrete space $\omega$, and discussion of this remainder takes up a sizable chunk of a book that was published back in 1960 [8]. It is remarkable that one of the most basic questions about it is still unsolved:

Problem 1. Is it consistent that $\omega^{*}$ is homeomorphic to $\omega_{1}^{*}$ ?

Here $\omega_{1}^{*}$ refers to the Cech-Stone remainder of a discrete space of cardinality $\omega_{1}$. What makes this problem all the more remarkable is that if we put any other pair of distinct infinite cardinals for $\left\{\omega, \omega_{1}\right\}$, even if one of this pair is one of the members of the new pair, the answer is negative. Moreover, this has been known since the late 1970's. Since most of the research that established this and other nontrivial facts detailed later was done by Polish and Czech mathematicians [2] [6] [7], I decided to break with the usual American custom and use the expression "Čech-Stone" in place of "Stone-Čech."

An interesting alternative formulation of Problem 1 in ZFC is:

Problem 1'. Is it consistent that the Boolean algebras $\mathcal{P}(\omega) /$ fin and $\mathcal{P}\left(\omega_{1}\right) /\left[\omega_{1}\right]^{<\omega}$ are isomorphic?

In the absence of the Axiom of Choice (AC) the two problems are not equivalent: what passes for the Čech-Stone remainders could be empty, while the quotient algebras are both uncountable. It would be interesting if the Boolean Algebra version had a positive answer in ZF while the answer to both versions is negative in ZFC. While our primary interest is what happens in ZFC, I will be making remarks about what to watch out for if $A C$ is not assumed. The theory would have a varying flavor depending on what weakenings of $A C$ are assumed. Three natural weakenings are: (1) the Boolean Prime Ideal Theorem, which assures that every discrete space has a Čech-Stone compactification; (2) the existence of right inverses to the two quotient maps; and (3) the axiom of dependent choices (DC), which implies the Countable AC (= AC for countable collections of sets).

2000 Mathematics Subject Classification. 54A25, 54A35, 54D20, 54D45, 54G20.

Key words and phrases. Čech-Stone remainder, 
Three other weakenings of AC dovetail well with Problem 1. Let $\operatorname{WAC}(\kappa, \lambda)$ stand for the axiom that there is a choice function for collections of $\leq \kappa$ sets, each of cardinality $\leq \lambda)$. Then $\operatorname{WAC}\left(2^{\omega_{1}}, \omega_{1}\right)$ implies $(2)$ above; WAC $\left(2^{\omega}, \omega\right)$ implies that the quotient map from $\mathcal{P}(\omega)$ to $\mathcal{P}(\omega) /$ fin has a right inverse; and we will see some proofs which go through if $\{\kappa, \lambda\} \subset\left\{\omega, \omega_{1}\right\}$. An interesting but farfetched scenario is that of Problem 1 having a Yes answer in the absence of (2), yet for the quotient map from $\mathcal{P}(\omega)$ to $\mathcal{P}(\omega) /$ fin to have a right inverse.

The following natural variation on Problem 1 is also unsolved:

? 1002 Problem 2. Is it consistent for $U(\kappa)$ and $U(\lambda)$ to be homeomorphic for different $\kappa, \lambda$ ?

Here $U(\kappa)$ stands for the set of uniform ultrafilters on $\kappa$ - those of which every member is of cardinality $\kappa$. It is very easy to show that there is no homeomorphism if $c f(\kappa) \neq c f(\lambda)$ (see Theorem ??) but even the case $\kappa=\omega, \lambda=\aleph_{\omega}$ has resisted all attempts at a solution. The Boolean algebra version of Problem 2 is left as an exercise for the reader; facts from Section 2 make this exercise trivial.

The conventional wisdom is that Problems 1 and 2 have negative answers, so I could easily have worded Problem 1, "Is it a theorem of ZFC that $\omega^{*}$ is not homeomorphic to $\omega_{1}^{*}$ ?" and used a similar wording for Problem 2. However, I am recommending that we treat the claim that $\omega^{*}$ and $\omega_{1}^{*}$ ARE homeomorphic as an axiom, the way Rothberger boldly treated $\mathfrak{p}>\omega_{1}$ even though all the evidence then available (including Godel's proof of consistency of $\mathrm{CH}$ ) suggested it was false. Recall also how Bing, unaware of Rothberger's research, published an example of a nonmetrizable separable normal Moore space on the assumption that there exists a Q-set, known to be contradicted by the natural-seeming axiom $2^{\omega}<2^{\omega_{1}}$; and how Mary Ellen Rudin published the first example of a Dowker space assuming the existence of a Souslin tree, also not known at the time to be consistent. Accordingly I formulate:

$\operatorname{AXIOM~} \Omega: \omega^{*}$ is homeomorphic to $\omega_{1}^{*}$.

Nowadays people are reluctant to assume axioms so boldly, except perhaps in the case of large cardinal axioms, but there are certain advantages to this approach. It encourages researchers to publish consequences of the axiom in the optimistic hope that some day the axiom may turn out to be consistent, so that if the breakthrough does happen, we will have a whole body of different statements known to be simultaneously consistent. On the other hand, if the axiom should turn out to be false, the proof that this is so will probably be a proof by contradiction, building upon consequences of the axiom that are already known.

Clearly, AXIOM $\Omega$ implies $2^{\omega}=2^{\omega_{1}}$ : the weights of $\omega^{*}$ and $\omega_{1}^{*}$ are $2^{\omega}$ and $2^{\omega_{1}}$ respectively. Two other easy consequences of AXIOM $\Omega$ are that there is a complete $\omega_{1}$-tower (Theorem 2.2) and, in contrast, that there is a Q-set. The contrast is heightened in both directions in Section 3, by theorems 3.1 and 3.3 
respectively. A great many natural questions about the implications of AXIOM $\Omega$ remain unanswered; a short list is given in Section 5 .

We assume AC except where explicitly stated otherwise. Lower-case Gothic letters designate small uncountable cardinals [5], [12].

\section{Some Basics}

We recall some basic facts about the Čech-Stone compactifications of discrete spaces. The underlying set for $\beta D$, where $D$ is a discrete space, is the set of all ultrafilters on $D$. A base for the topology is the collection of all sets of the form $[A]=\{p \in \beta D: A \in p\}$. This makes $\beta D$ into a compact space, which is 0 -dimensional because each $[A]$ is clopen. The discrete space $D$ is identified with the set of principal ultrafilters and is the dense set of isolated points of $\beta D$. The Cech-Stone remainder $\beta D-D$ is designated $D^{*}$, and $[A] \backslash A(=[A] \backslash D)$ is designated $A^{*}$. The sets of the form $A^{*}$ thus form a base for the relative topology on $D^{*}$.

The collection $C O(X)$ of clopen sets of any topological space $X$ is a Boolean algebra under the usual operations of $\cup, \cap$, and complementation. The following facts are well known, easy to prove, and useful; for instance, (b) clearly implies the equivalence of Problems 1 and 1.

(a) The unary operation $[\cdot]$ is a Boolean algebra isomorphism from $\mathcal{P}(D)$ to $C O(\beta D)$.

(b) The unary operation * is a Boolean algebra homomorphism from $\mathcal{P}(D)$ onto $C O\left(D^{*}\right)$, whose kernel is $[D]^{<\omega}$.

(c) For each infinite cardinal $\kappa \leq|D|$, the set of ultrafilters whose smallest members are of cardinality $<\kappa$ is a dense open subspace of $D^{*}$. Therefore, $U(D)$ is a nowhere dense closed subspace of $\beta D$.

(d) If we define $A^{\wedge}$ as $\{[A] \cap U(D): A \subset D\}$ then ^ is a Boolean algebra homomorphism from $\mathcal{P}(D)$ onto $C O(U(D))$, whose kernel is $[D]^{<|D|}$.

(e) If $X$ and $Y$ are compact 0 -dimensional spaces and $\phi: X \rightarrow Y$ is continuous, and $\phi^{\leftarrow}: C O(Y) \rightarrow C O(X)$ is defined by $\phi^{\leftarrow}(K)=\phi^{-1}(K)$ then $\phi^{\leftarrow}$ is a Boolean algebra homomorphism. Moreover, $\phi^{\leftarrow}$ is injective iff $\phi$ is surjective, and vice versa.

(f) A continuous bijection between compact Hausdorff spaces is a homeomorphism. Consequently, $\phi^{\leftarrow}$ is an isomorphism iff $\phi$ is a homeomorphism.

A good understanding of $\omega^{*}$ and $\omega_{1}^{*}$ calls for skill in shuttling back and forth between $\mathcal{P}(D)$ and $C O\left(D^{*}\right)$, using the * operation to "go upstairs" from $D$ to $D^{*}$ and implicitly using various choice functions to "go downstairs" by labeling clopen subsets of $D^{*}$ as $A^{*}$, etc. This is where the various weakenings of AC come in. [In their absence, we translate topological language on $\omega^{*}$ by using the natural correspondence between open sets and ideals, and between clopen sets and elements (or principal ideals).] 
A similar shuttle works for $\mathcal{P}(D)$ and $U(D)$. We see it operating in the proof of the following theorem [4].

Theorem 2.1. If $c f(\kappa) \neq c f(\lambda)$, then $U(\kappa)$ and $U(\lambda)$ are not homeomorphic.

Proof. Let $c f(\kappa)<c f(\lambda)$. Partition $\lambda$ into $c f(\kappa)$ sets $A_{\alpha}$ of cardinality $\lambda$. The

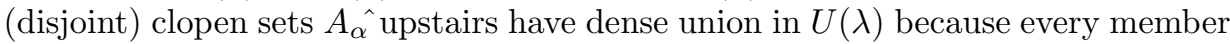
of $[\lambda]^{\lambda}$ meets some $A_{\alpha}$ in a set of size $\lambda$. On the other hand, no family of $c f(\kappa)$ disjoint clopen subsets $B_{\alpha} \hat{\text { of }} U(\kappa)$ is dense in $U(\kappa)$ : there is a $\kappa$-element subset of $\kappa$ which meets $B_{\alpha}$ in a set of cardinality $|a|$ and is disjoint from all earlier $B_{\beta}$.

In analyzing $\omega_{1}^{*}$ the dense open subspace $S\left(\omega_{1}\right)=\omega_{1}^{*} \backslash U\left(\omega_{1}\right)$, known as the space of subuniform ultrafilters, plays an important role. It is the union of the ascending $\omega_{1}$-chain $\left\{\alpha^{*}: \alpha\right.$ is a countable limit ordinal $\}$ of clopen sets. In other words, it is what I call an $\omega_{1}$-oval:

Definition. A union of a chain of clopen sets in a Čech-Stone remainder of a discrete space is an oval and is a $\kappa$-oval if the chain has cofinality $\kappa$.

In particular, the $\omega$-ovals are the cozero sets. The small uncountable cardinal $\mathfrak{t}$ can be characterized as the least $\kappa$ such there is a dense $\kappa$-oval in $\omega^{*}$. With this in mind it is easy to see:

Theorem 2.2. AXIOM $\Omega$ implies $\mathfrak{t}=\omega_{1}$.

The Boolean algebra version is that there is an ideal generated by an $\omega_{1}$-chain in $\mathcal{P}(\omega) /$ fin that meets every nonzero ideal.

Another shuttle goes between $\mathcal{P}(D)$ (or $C O\left(D^{*}\right)$ ) and $\mathcal{P}(\omega \times \omega)$. I call it the $R H$ Transfer in honor of Rothberger and Hechler, who made good use of it.

Definition. Let $\mathcal{A}=\left\{A_{n}: n \in \omega\right\}$ be a family of subsets of $\omega$ such that $A_{n}^{\#}=$ $A_{n} \backslash \bigcup_{i=0}^{n-1} A_{i}$ is infinite for all $n$. An $R H$ transfer of $\mathcal{A}$ to $\omega \times \omega$ is a bijection $\psi: \omega \rightarrow \omega \times \omega$ which distributes the elements of $\omega \backslash \bigcup_{n=0}^{\infty} A_{n}$ into the bottom row $\omega \times\{0\}$, and sends $A_{n}^{\#}$ into the $(n+1)$ st column $\{n\} \times \omega$.

In an $\mathrm{RH}$ transfer, subsets of $\omega$ that are almost disjoint from all the $A_{n}$ are characterized by their images being dominated by the graph of a function. The transfer and the definition of the function can all be defined in ZF, taking advantage of the listing of $\mathcal{A}$ and the well-ordering on $\omega$. It is when we combine the transfers with moves downstairs that some form of $A C$ is required. The following simple theorem [9] only requires $\operatorname{WAC}(\omega, \omega)$ in a move downstairs followed by composing one $\mathrm{RH}$ transfer with the inverse of another, followed by a move upstairs.

Theorem 2.3. Any two $\omega$-ovals in $\omega^{*}$ are homeomorphic; moreover, there is a permutation of $\omega$ whose extension to $\beta \omega$ is a homeomorphism taking one to the other. 


\section{Some CONSEQUences of AXIOM $\Omega$}

There is some confusion about whether "Q-set" is understood to include "uncountable," so I have suggested extending the usual list of Gothic-letter small cardinals to include $\mathfrak{q}$. The trouble is, there are two natural and useful rivals for what $\mathfrak{q}$ could designate. So I recommend using subscripts, as follows:

$\mathfrak{q}_{0}=$ the least cardinal $\kappa$ for which there is a set of reals of size $\kappa$ that is not a Q-set.

$\mathfrak{q}_{1}=$ the least cardinal $\kappa$ for which no set of reals of size $\kappa$ is a Q-set.

Theorem 3.1. AXIOM $\Omega$ implies $\mathfrak{q}_{1}>\omega_{1}$.

This theorem is a corollary of a much stronger theorem mentioned (but not proved) in [10]. Call a family $\mathcal{A}$ of $\omega_{1}$-many denumerable subsets of $\omega$ a strong $Q$-sequence if every 2-coloring of the members of $\mathcal{A}$ is uniformizable. This means that if $f_{A} \rightarrow\{0,1\}$ is given for each $A \in \mathcal{A}$, there is a function $f: \omega \rightarrow\{0,1\}$ such that $f(a)=f_{A}(a)$ for all but finitely many $a$ in each $A \in \mathcal{A}$. Clearly any strong Q-sequence is an $\mathrm{AD}$ family, by which I mean a collection of denumerable sets such that the intersection of any two is finite. We will see below how a Q-set of cardinality $\omega_{1}$ is intimately associated with the special case where each $f_{A}$ is constant.

Unlike $\mathfrak{q}_{1}>\omega_{1}$, the existence of a strong Q-sequence does not follow from $M A_{\omega_{1}}$ and indeed is incompatible with it [10]. In contrast:

Theorem 3.2. AXIOM $\Omega$ implies there is a strong Q-sequence.

Proof. For each countable limit ordinal $\alpha$ let $A_{\alpha}=[\alpha, \alpha+\omega)$. Obviously, every 2-coloring (indeed every coloring!) of the individual $A_{\alpha}$ is uniformizable. Upstairs in $\omega_{1}^{*}$, uniformizability of every 2-coloring translates into the following: for each choice of clopen $C_{\alpha} \subset A_{\alpha}^{*}$ there is a clopen $K$ such that $K \cap A_{\alpha}=C_{\alpha}$ for all $\alpha$. [Just let $C_{\alpha}$ be the remainder of the support of $f_{A_{\alpha}}$, etc.] Assuming AXIOM $\Omega$ we shuttle over to $\omega^{*}$ with a homeomorphism $\psi$. The images of the $A_{\alpha}$ move downstairs to an $\mathrm{AD}$ family on $\omega$ which is easily seen to be a strong Q-sequence by a translation like that above.

Among the many statements equivalent to $\mathfrak{q}_{1}>\omega_{1}$ is the existence of a separable nonmetrizable normal Moore space, as well as the existence of the special case where the Moore space is locally compact and its set of nonisolated points is a closed discrete space; see [11] and its references in Section II. This special case has a nice alternative characterization as a normal uncountable $\Psi$-like space:

Definition. A $\Psi$-like space is a locally compact, locally countable space $X$ in which $\omega$ is a dense set of isolated points and $X \backslash \omega$ is closed discrete.

We can associate an AD family $\mathcal{A}$ on $\omega$ with the nonisolated points of a $\Psi$-like space $X$, with each $A \in \mathcal{A}$ associated with a point $p_{A}$ such that $A \cup\left\{p_{A}\right\}$ is a compact open neighborhood of $p_{A}$. Normality of $X$ then translates to uniformizability of every 2 -coloring of $\mathcal{A}$ in which each $f_{A}$ is constant. In this way, Theorem 3.1 is made to follow from Theorem 3.2. 
Rothberger showed that $\mathfrak{q}_{0} \leq \mathfrak{b}$. Since $\mathfrak{b} \leq \mathfrak{d}$, the following theorem shows that AXIOM $\Omega$ implies $\mathfrak{q}_{0}=\omega_{1}$.

Theorem 3.3. AXIOM $\Omega$ implies $\mathfrak{d}=\omega_{1}$.

The proof of this theorem in [4] starts with the assumption that $\kappa^{*}$ is homeomorphic to $\omega^{*}$ (where $\kappa$ is regular uncountable). It makes a downstairs move that implicitly uses $\operatorname{WAC}\left(\omega_{1}, \omega_{1}\right)$ and then explicitly constructs a $\kappa$-scale in $\left({ }^{\omega} \omega,<^{*}\right)$, i.e., a cofinal family of order type $\kappa$ under the order $<^{*}$ of strict eventual domination. Recall that $\mathfrak{d}$ is the least (uncountable) cardinality of a cofinal family in this order. Thus Theorem 3.3 is established.

The case of general $\kappa$ is then made in [4] to lead to a contradiction in evey case except $\kappa=\omega_{1}$, as part of a sequence of proofs that culminates in the theorem mentioned in the paragraph following Problem 1.

There is a topological route to Theorem 3.3 via the following theorem:

Theorem 3.4. Let $\kappa$ be a regular cardinal. The following are equivalent.

(1) There is a $\kappa$-scale.

(2) The exterior of some (hence every) $\omega$-oval in $\omega^{*}$ is a $\kappa$-oval.

(3) there is an $\omega$-oval $E$ and a family $\mathcal{C}$ of $\kappa$ disjoint clopen sets in $\omega^{*}$ such that every clopen set containing $E$ also contains all but $<\kappa$ members of $\mathcal{C}$, but also every subfamily of $<\kappa$ members of $\mathcal{C}$ is missed by some clopen set containing $E$.

This theorem needs only ZF for the forward implications but the reverse implications both seem to require moves downstairs utilizing $\operatorname{WAC}(\kappa, \omega)$. The topological proof of Theorem 3.3 is finished by finding a pair $E, \mathcal{C}$ in $\omega_{1}^{*}$ that answers to the description in (3) of Theorem 3.4, with $\kappa=\omega_{1}$. The following pair needs nothing beyond ZF:

$$
E=\bigcup_{n=0}^{\infty} A_{n}^{*} \text { where } A_{n}=\{\alpha+n: \alpha \in \Lambda \cup\{0\}\}
$$

(where $\Lambda$ stands for the set of countable limit ordinals) and

$$
\mathcal{C}=\left\{C_{\alpha}: \alpha \in \Lambda\right\} \text { where } C_{\alpha}=[\omega \cdot \alpha, \omega \cdot(\alpha+1))^{*}
$$

In particular, if $K$ is a clopen set that meets uncountably many $C_{\alpha}$, and $K=B^{*}$, then $B$ meets uncountably may intervals $[\omega \cdot \alpha, \omega \cdot(\alpha+1))$ and so it meets some $A_{n}$ in an uncountable set. Therefore, $K \cap E \neq \emptyset$, and any clopen set that contains $E$ consequently must contain all but countably many $C_{\alpha}$. Since we only moved downstairs with $K$, this much is true in ZF as long as one substitutes, if necessary, members of $\mathcal{P}\left(\omega_{1}\right) /\left[\omega_{1}\right]^{<\omega}$ for the clopen sets.

In contrast to this explicit example, the modification of (2) which puts $\omega_{1}^{*}$ in place of $\omega^{*}$ is actually equivalent to $\mathfrak{d}=\omega_{1}$ as will be explained in the next section. This may be behind the fact that I have been unable to show (3) implies (2) without going downstairs. 
Theorem 3.5. AXIOM $\Omega$ implies that there is a dense $\omega_{1}$-oval in $\omega^{*}$ whose complement does not contain any P-points.

There do exist models where this consequence of AXIOM $\Omega$ holds. In fact, in [13, 7.15] a proof attributed to Mary Ellen Rudin shows how the set $Z$ of ultrafilters on $\omega$ which do not contain any sets of density 0 is nowhere dense in $\omega$ and is a $\mathrm{P}$-set without P-points of $\omega^{*}$. Under $\mathrm{CH}$, the complement of $Z$ is an $\omega_{1}$-oval.

The key to Theorem 3.5 is that no uniform ultrafilter on $\omega_{1}$ is a P-point of $\omega_{1}^{*}$ : take a sequence of partitions $P_{n}$ of $\omega_{1}$ into $2^{n}$ uncountable pieces such that the common refinement of $P_{n}$ is the partition into singletons. Thus, in $\omega_{1}$, the subspace $U\left(\omega_{1}\right)$ fits the description of the complement.

Problem 3. Is there a model of $\mathfrak{d}=\omega_{1}$ in which there is a strong Q-sequence and also a dense oval as described in Theorem 3.5?

\section{ImPLiCATIONS FOR $\omega_{1}^{*}$}

There is a variation on $\mathrm{RH}$ transfer for $\omega_{1}$ that helps with the analysis of $\omega$ ovals in $\omega_{1}^{*}$. Let $\mathcal{A}=\left\{A_{n}: n \in \omega\right\}$ be a family of subsets of $\omega_{1}$ such that $A_{n}^{\#}=A_{n} \backslash \bigcup_{i=0}^{n-1} A_{i}$ is uncountable for all $n$. An $R H$-like transfer of $\mathcal{A}$ to $\omega_{1} \times \omega_{1}$ is a bijection $\psi: \omega_{1} \rightarrow \omega \times \omega_{1}$ which does one of two things, depending on whether $A_{\infty}=\omega_{1} \backslash \bigcup_{n=0}^{\infty} A_{n}$ is countable or uncountable. If it is countable, $\psi$ distributes the elements of $\omega_{1} \backslash \bigcup_{n=0}^{\infty} A_{n}$ into the bottom row $\omega \times\{0\}$, and sends $A_{n}^{\#}$ into the $(n+1)$ st column $\{n\} \times \omega_{1}$. If $A_{\infty}$ is uncountable, $\psi$ sends it onto $\{0\} \times \omega_{1}$ and $A_{n}^{\#}$ onto $\{n+1\} \times \omega_{1}$.

This transfer is good for analyzing $\omega$-ovals in $\omega_{1}^{*}$ that meet $U\left(\omega_{1}\right)$ in a noncompact subset. The first case represents ovals whose closure contains all of $U\left(\omega_{1}\right)$. If the exterior of such an oval is also an oval, then there is an almost-ascending sequence $\left\{A_{\alpha}: \alpha \in \omega_{1}\right\}$ of countable subsets of $\omega \times \omega_{1}$ such that every set that is almost disjoint from the columns of $\omega \times \omega_{1}$ is a subset of some $A_{\alpha}$. This implies $\mathfrak{d}=\omega_{1}$, as a look at the traces of the $A_{\alpha}$ on $\omega \times \omega$ shows. The converse is also easy for those used to the arguments in [5] involving $\beta$ and $\mathfrak{d}$. In the second case, where $A_{\infty}$ is uncountable, one looks at sets of the form $A_{\alpha} \cup A_{\infty}$ to arrive at the same conclusions. Also, $\omega$-ovals which do not meet $U\left(\omega_{1}\right)$ can be encapsuled in the remainder of a countable set; then, if $\mathfrak{d}=\omega_{1}$ we get the conclusion that every $\omega$-oval in $\omega_{1}^{*}$ has an $\omega_{1}$-oval exterior. Of course, AXIOM $\Omega$ gives the same conclusion even more easily, thanks to Theorems 2.3 and 3.3. The former theorem also shows (1) implies (5) in the following theorem, and together with the modified $\mathrm{RH}$ transfer in this section it easily implies (4) is equivalent to (5). The other implications, all of which are very easy, are shown in [4].

Theorem 4.1. The following are equivalent.

(1) AXIOM $\Omega$

(2) Any two nonempty clopen subsets of $\omega_{1}^{*}$ are homeomorphic.

(3) There is an autohomeomorphism of $\omega_{1}^{*}$ that does not take $U\left(\omega_{1}\right)$ to itself. 
(4) There is an autohomeomorphism of $\omega_{1}^{*}$ such that $U\left(\omega_{1}\right)$ is disjoint from its image.

(5) For any two $\omega$-ovals in $\omega_{1}^{*}$, there is an autohomeomorphism of $\omega_{1}^{*}$ taking one to the other.

We call an autohomeomorphism of $\omega^{*}$ or $\omega_{1}^{*}$ nontrivial if it cannot be induced by a 1-1 function from $\omega\left[\right.$ resp. $\left.\omega_{1}\right]$ to itself.

Corollary 1. AXIOM $\Omega$ implies that $\omega_{1}^{*}$ has nontrivial autohomeomorphisms.

? 1004 Problem 4. Does AXIOM $\Omega$ imply that $\omega^{*}$ has nontrivial autohomeomorphisms?

One might think that there are bijections from $\omega_{1}$ to itself whose effect on $\omega_{1}^{*}$ cannot be mimicked by functions from $\omega$ to itself acting on $\omega^{*}$, but appearances may be deceiving. The search for bijections without mimics is especially challenging in models where there are strong Q-sequences.

\section{Some More open PRoblems ABout AXIOM $\Omega$}

Of the endless questions one might ask about the implications of $\operatorname{AXIOM~} \Omega$, the following seem especially natural to me:

? 1005 Problem 5. Does Axiom $\Omega$ place any restrictions on $2^{\omega}$ besides the usual one (it cannot have countable cofinality) and the denial of $\mathrm{CH}$ ?

? 1006 Problem 6. Does Axiom $\Omega$ imply that there are (or are not?!) P-points in $\omega^{*}$ ?

Theorem 3.5 shows that every P-point of $\omega_{1}^{*}$ is in $S\left(\omega_{1}\right)$.

? 1007 Problem 7. Does Axiom $\Omega$ have any implications for the small uncountable cardinals $\mathfrak{a}, \mathfrak{i}, \mathfrak{r}, \mathfrak{u}$ ?

? 1008 Problem 8. Does Axiom $\Omega$ negate \&? "stick"?

Axiom "stick" states that there is a family $\mathcal{A}$ of $\omega_{1}$ countable subsets of $\omega_{1}$ such that every uncountable subset of $\omega_{1}$ contains some member of $\mathcal{A}$, while $\boldsymbol{\beta}$ adds the condition that $\mathcal{A}$ is a ladder system.

Note that Axiom $\Omega+$ "stick" implies $\mathfrak{r}=\omega_{1}$, since $\mathfrak{r}$ is the least cardinality of a $\pi$-base for a free ultrafilter on $\omega$. [A family of sets witnessing "stick" is a $\pi$-base for every uniform ultrafilter on $\omega_{1}$.]

? 1009 Problem 9. Does Axiom $\Omega$ imply that there is a family of more than $\omega_{1}$ disjoint clopen sets in $\omega_{1}^{*}$, each of which meets $U\left(\omega_{1}\right)$ ?

If the answer to this problem is Yes, then so is the answer to Problem 8. In contrast, if Axiom $\Omega$ implies all disjoint clopen families of cardinality $\mathfrak{c}$ have (all but $<\mathfrak{c}$ ) members missing $U\left(\omega_{1}\right)$, we must look elsewhere than the density example for a mimic of $U\left(\omega_{1}\right)$ in $\omega^{*}$, because that nowhere dense P-set $Z$ is met by a family of c-many disjoint clopen subsets of $\omega^{*}$. To see this, partition $\omega$ into two subsets, such that in both of them the ratio of numbers $<n$ to $n$ gets arbitrarily close to both 0 and 1 . Repeat this process countably many times, and diagonalize to get $\mathfrak{c}$-many almost disjoint subsets of $\omega$ in which this same phenomenon happens. Each one is in some member of $Z$. 
Problem 10. Does Axiom $\Omega$ have any implications for the cardinals in Cichon's diagram [12] that are not below $\mathfrak{d}$ ?

Problem 11. Does Axiom $\Omega$ imply that there are no $\omega_{2}$-ovals in $\omega^{*}$ ?

Problem 12. Does Axiom $\Omega$ imply that there is an $\omega_{1}$-oval in $\omega^{*}$ whose exterior is also an $\omega_{1}$-oval?

In [3] it is shown (in effect) that $\mathfrak{t}=\omega_{1}$ is equivalent to there being a pair of disjoint $\omega_{1}$-ovals in $\omega^{*}$ whose union is dense in $\omega^{*}$, but there are models of $\mathfrak{t}=\omega_{1}$ where neither can be the exterior of the other. I am unaware of any such models where $\mathfrak{d}=\omega_{1}$, however.

If $\boldsymbol{q}$ holds, the subspace $S\left(\omega_{1}\right)$ of $\omega_{1}^{*}$ can be split into two disjoint $\omega_{1}$-ovals, each of which has all of $U\left(\omega_{1}\right)$ in its closure, making each one the exterior of the other. On the other hand, this is impossible if what is called $(*)$ in [1] holds. But in this latter case there may be ways of constructing disjoint $\omega_{1}$-ovals inside $A^{*}$ for some countable $A$, each of which is the exterior in $A^{*}$ of the other; this is easy to do under $\mathrm{CH}$, which is compatible with $(*)$. Then the union of one oval with the complement of $A^{*}$ in $\omega_{1}^{*}$ is an $\omega_{1}$-oval whose exterior in $\omega_{1}^{*}$ is the other oval.

\section{Notes on Problem 2}

Comparatively little research has been done on the implications of a Yes answer to Problem 2. Unlike with Problem 1, there is no end of pairs $\kappa, \lambda$ that are candidates for an affirmative answer. Here we content ourselves with a few observations about the pair $\omega, \aleph_{\omega}$. Most of what we will say carries over to any other pair that is not eliminated by Theorem 2.1 .

First, an argument similar to the construction of a Bernstein set shows that if $\mathcal{K}$ is a collection of $\kappa$ sets of cardinality $\kappa$, then there is a pair of disjoint sets which meets each one in a set of cardinality $\kappa$. So if $U\left(\aleph_{\omega}\right)$ is homeomorphic to $U(\omega)\left(=\omega^{*}\right)$, the reaping number $\mathfrak{r}$ is $\geq \aleph_{\omega+1}$.

The other observations have to do with the variety of dense ovals in $U\left(\aleph_{w}\right)$, summarized in the following theorem. In any model where it is homeomorphic to $U(\omega)\left(=\omega^{*}\right)$, we get the same variety in $\omega^{*}$, in marked contrast to the little we know about ovals in $\omega^{*}$ if AXIOM $\Omega$ holds (see Problem 11).

Theorem 6.1. $U\left(\aleph_{\omega}\right)$ has dense $\kappa$-ovals for all $\kappa$ such that $\omega<\kappa<\aleph_{\omega}$ and also $\kappa=\mathfrak{b}$ and also for all regular $\kappa$ between $\aleph_{\omega}$ and $\min \left\{c f\left[\aleph_{\omega}\right]^{\omega}, \aleph_{\omega_{1}}\right\}$.

For $\kappa=\omega_{n}(n>0)$, use $\aleph_{\omega} \times \omega_{n}$ and let $C_{\alpha}=\aleph_{\omega} \times \alpha$; a cofinality argument shows that every subset of $\aleph_{\omega} \times \omega_{n}$ of cardinality $\aleph_{\omega}$ meets some $C_{\alpha}$ in a set of cardinality $\aleph_{\omega}$. The remainders of the $C_{\alpha}$ in $U\left(A_{\omega}\right)$ union up to a dense $\kappa$-oval.

For regular $\kappa$ from $\aleph_{\omega}$ to the minimum of $c f\left[\aleph_{\omega}\right]^{\omega}$ and $\aleph_{\omega_{1}}$, use the powerful result of pcf theory that there are subsequences of $\left\{\aleph_{n}: n \in \omega\right\}$ where the product has a scale of the desired length. Let $C_{\alpha}$ be the part of $\aleph_{\omega} \times \omega$ below the graph of $f_{\alpha}$. 
For $\kappa=\mathfrak{b}$ use $A_{\omega} \times \omega$ and functions $f_{\alpha} \alpha<\mathfrak{b}$ that are constant on $\left[\omega_{n}, \omega_{n+1}\right)$ and nondecreasing, and are well-ordered by the order of eventual domination. Here too, let $C_{\alpha}$ be the part of $\aleph_{\omega} \times \omega$ below the graph of $f_{\alpha}$.

This last argument works for any $\kappa$ for which there is a $<{ }^{*}$-unbounded $<*$-wellordered family of increasing functions of cofinality $\kappa$ in $\omega^{\omega}$.

\section{REFERENCES}

[1] Abraham, U. and Todorčević, S., Partition properties of $\omega_{1}$ compatible with CH, Fund. Math. 152 (1997) 165-181.

[2] Balcar, B. and Frankiewicz, R., To distinguish topologically the spaces $m^{*}$ II, Bull. Acad. Polon. Sci. Sr. Sci. Math. Astronom. Phys. 26 (1978), no. 6, 521-523.

[3] Blasczyk, A. and Szymański, A. Hausdorff's gaps versus normality, Bull. Acad. Polon. Sci. Sér. Sci. Math. 30 (1982), no. 7-8, 371-378.

[4] Comfort, W. W., Compactifications: recent results from several countries, Topology Proc. 2 (1977), no. 1, 61-87 (1978).

[5] van Douwen, E.K., The integers and topology, in: Handbook of Set-Theoretic Topology, K. Kunen and J. Vaughan ed., North-Holland, 1984, pp. 111-167.

[6] Frankiewicz, R. To distinguish topologically the space $m^{*}$. Bull. Acad. Polon. Sci. Sr. Sci. Math. Astronom. Phys. 25 (1977), no. 9, 891-893. 54D40

[7] _ Assertion $Q$ distinguishes topologically $\omega^{*}$ and $\mathfrak{m}^{*}$ when $\mathfrak{m}$ regular and $\mathfrak{m}>\omega$, Colloq. Math. 38 (1977/78), no. 2, 175-177.

[8] Gillman, L., and Jerison, M., Rings of Continuous Functions, van Nostrand, 1960.

[9] Hechler, S. H., A dozen small uncountable cardinals, in: TOPO 72: General Topology and its Applications, Lecture Notes in Mathematics No. 378, Springer-Verlag, 1974, pp. 207-218.

[10] Steprans, J., Strong Q-sequences and variations on Martin's Axiom, Canad. J. Math. 37 (1985) no. 4, 730-746.

[11] Tall, F. D., Normality versus collectionwise normality, in: Handbook of Set-Theoretic Topology, K. Kunen and J. Vaughan ed., North-Holland (1984) 685-732.

[12] Vaughan, J., Small uncountable cardinals and topology. With an appendix by S. Shelah, in: Open problems in topology, Jan van Mill and George M. Reed, ed., North-Holland, Amsterdam, 1990, pp. 195-218.

[13] Walker, R. C., The Stone-Čech compactification, Ergebnisse der Mathematik und ihrer Grenzgebiete, Band 83, Springer-Verlag, New York-Berlin, 1974.

Definition. A union of a chain of clopen sets is an oval and is a $\kappa$-oval if the chain has cofinality $\kappa$.

Department of Mathematics, LeConte College, 1523 Greene Street, University of South Carolina Columbia, SC 29208

E-mail address: nyikos@math.sc.edu 